\title{
Análise da produção científica sobre a tecnologia e a inovação no agronegócio
}

\author{
Analysis of scientific production on technology and innovation in agribusiness \\ Análisis de producción científica sobre tecnología e innovación em agronegocios
}

Recebido: 09/05/2021 | Revisado: 13/05/2021 | Aceito: 13/05/2021 | Publicado: 15/05/2021

Isabel Cristina dos Santos

ORCID: https://orcid.org/0000-0001-5505-5234 Universidade Municipal de São Caetano do Sul, Brasil

E-mail: isa.santos.sjc@gmail.com

Glaucia Bambirra Silveira

ORCID: https://orcid.org/0000-0003-2836-1265 Universidade Municipal de São Caetano do Sul, Brasil E-mail: glauciabambirra@gmail.com

Renato Emanuel Gomes da Silva

ORCID: https://orcid.org/0000-0001-6438-7142

Universidade Municipal de São Caetano do Sul, Brasil E-mail: renatoemmanuel@hotmail.com

\begin{abstract}
Resumo
O estudo visa apresentar a literatura mais recente sobre o tema inovação e tecnologia aplicadas ao agronegócio. O trabalho consistiu em realizar uma análise dos artigos científicos publicados no banco de dados da Web of Science no período de 2016 a 2020. Trata-se de uma pesquisa exploratória, de abordagem quantitativa, com a utilização da Teoria do Enfoque Meta-analítico Consolidado, que proporciona uma abordagem integradora de revisão da literatura. Os resultados apontaram que 2.427 artigos científicos, nacionais e internacionais, em vários idiomas e em diversas áreas do conhecimento, adotaram abordagem simultânea dos descritores inovação, tecnologia e agronegócio, no período de 2016 a 2020. Dentre esses estudos, destacam-se 104 artigos elaborados por pesquisadores brasileiros. A Empresa Brasileira de Pesquisa Agropecuária, Universidade de São Paulo e a Universidade Estadual Paulista foram as instituições brasileiras que mais se destacaram nas produções acadêmicas, apresentando trabalhos compartilhados com organizações brasileiras e estrangeiras, o que contribuiu para a difusão do conhecimento no tema inovação e tecnologia no agronegócio.
\end{abstract}

Palavras-chave: Tecnologias digitais; Inovação tecnológica; Agronegócio; Enfoque meta-analítico.

\begin{abstract}
The study aims to present the most recent literature on the theme of innovation and technology applied to agribusiness. The work consisted of carrying out an analysis of the scientific articles published in the Web of Science database in the period from 2016 to 2020. This is an exploratory research, with a quantitative approach, using the Consolidated Meta-Analytical Approach Theory, which provides an integrative approach to literature review. The results showed that 2,427 scientific articles, national and international, in several languages and in different areas of knowledge, adopted a simultaneous approach of the keywords innovation, technology and agribusiness, in the period from 2016 to 2020. Among these studies, 104 articles were highlighted by Brazilian researchers. The Brazilian Agricultural Research Corporation, Universidade de São Paulo and Universidade Estadual Paulista were the Brazilian institutions that stood out the most in academic production, presenting works shared with Brazilian and foreign organizations, which contributed to the dissemination of knowledge on innovation and technology in agribusiness sector.
\end{abstract}

Keywords: Digital technologies; Technological innovation; Agribusiness; Meta-analytical approach.

\section{Resumen}

El estudio tiene como objetivo presentar la literatura más reciente sobre el tema de la innovación y la tecnología aplicadas a la agroindustria. El trabajo consistió en realizar un análisis de los artículos científicos publicados en la base de datos de Web of Science en el período de 2016 a 2020. Se trata de una investigación exploratoria, con enfoque cuantitativo, utilizando la Teoría del Enfoque Metaanalítico Consolidado, que proporciona una enfoque integrador de la revisión de la literatura. Los resultados mostraron que 2.427 artículos científicos, nacionales e internacionales, en varios idiomas y en diferentes áreas del conocimiento, adoptaron un abordaje simultáneo de los descriptores innovación, tecnología y agroindustria, en el período de 2016 a 2020. Entre estos estudios, 104 destacan los artículos elaborados por investigadores brasileños. La Corporación Brasileña de Investigación Agropecuaria, la Universidade 
de São Paulo y la Universidade Estadual Paulista fueron las instituciones brasileñas que más se destacaron en la producción académica, presentando trabajos compartidos con organizaciones brasileñas y extranjeras, que contribuyeron a la difusión del conocimiento sobre el tema de la innovación y la tecnología. en la agroindustria.

Palabras clave: Tecnologías digitales; Innovación tecnológica; Agroindustria; Enfoque metaanalítico.

\section{Introdução}

O Brasil é destaque na produção e exportação de alimentos, de forma que o agronegócio representa um setor importante na economia brasileira. A produção agropecuária, como complexo agroindustrial, pode ser dividida em quatro setores principais: instituições, indústria de insumos, comercializações e indústrias de processamento (Gonçalves et al., 2018). Além disso, integram o ecossistema do agronegócio as instituições e ensino e pesquisa e científicas.

A este propósito, o Sistema Brasileiro de Ciência e Tecnologia no setor agropecuário possui interações robustas com redes de pesquisa nacionais e internacionais e tem, na alta tecnologia aplicada, o valor agregado aos produtos e serviços, podendo servir como referência no desenvolvimento e implantação da pesquisa no setor do agronegócio, em nível mundial (Santos et al., 2020).

Nesse contexto, analisar as relações entre as universidades, as empresas, os agricultores e pecuaristas pode fortalecer o conhecimento sobre a transferência de conhecimento orientado para gerar tecnologia, inovação e soluções tecnológicas, que visam aumentar a produtividade e agregam valor aos negócios (Carayannis et al.,2018).

Cabe ressaltar que o conceito de agronegócio abarca todas as operações que envolvem desde a produção até o armazenamento, processamento e distribuição dos produtos agropecuários (Gonçalves et al.,2018), em um esforço de produção com segurança alimentar do Brasil para o mundo, que leva o país estar entre os maiores exportadores mundiais de alimentos (Moretti, 2020).

$\mathrm{Na}$ busca pela produtividade, a tecnologia encontrou terreno fértil para o desenvolvimento no agronegócio, especialmente no aumento da produção de alimentos, redução de custos, diminuição de perdas e aumento da produção agrícola, sem consequente aumento da área de plantio (Bernardo et al.,2018) e do trato animal, como a adaptação de cultivares para adoção de gramíneas de maior valor nutritivo.

Moretti (2020) atribui o crescimento do país na produção agropecuária como consequência de muita pesquisa, como ocorreu na revolução do Cerrado Brasileiro, onde as condições antes inóspitas para o plantio deram lugar a um mar verdejante e dourado da soja, entre outras culturas.

Por outro lado, os avanços tecnológicos ampliaram as formas de acesso ao conhecimento e às informações disponíveis sobre um determinado assunto, de forma que buscar o conhecimento pode ser um desafio para o pesquisador (Mariano e Rocha, 2017). Como uma etapa preliminar ao planejamento a pesquisa, o uso contínuo de técnicas que propiciem buscar o estado-da-arte no conhecimento científico relacionado ao tema, objeto do estudo é um recurso bastante útil para identificar a lacuna da pesquisa a ser desenvolvida e como o tema vem sendo debatido.

Este estudo de revisão sistemática da literatura pretende contribuir para identificar os principais temas efervescentes abordados pelos pesquisadores brasileiros que constam na base de dados Web of Science, as palavras-chaves mais utilizadas, as organizações e os periódicos com maior quantidade de artigos científicos publicados com a abordagem inovação e tecnologia no agronegócio com a finalidade de identificar as lacunas para desenvolvimento de novos estudos.

Ao mapear a produção dos pesquisadores nacionais e internacionais sobre inovação e tecnologia no agronegócio, este trabalho contribui para o aprimoramento das pesquisas cientificas e sua divulgação ao identificar os artigos mais citados, principais termos-chave utilizados pelos autores e os periódicos mais relevantes e que têm na temática da inovação no agronegócio um foco de interesse. Assim, trata-se de uma contribuição que apoia a escrita científica. 
Dessa forma, a presente pesquisa examina os estudos disponíveis sobre os temas inovação e tecnologia no agronegócio utilizando o banco de dados da Web of Science nos últimos cinco anos (2016 a 2020), englobando as diferentes áreas do conhecimento, visando relatar as principais contribuições de pesquisadores brasileiros.

\section{Revisão da Literatura}

Além de configurar-se como um ativo exportável com grande participação na balança comercial brasileira, a produção de alimentos contribui com uma parcela significativa da produção mundial (Haberli et al, 2017), especialmente em virtude da crescente demanda mundial por alimentos (Leite e Batalha, 2016), o que aumenta a pressão sobre os sistemas de produção alimentar (Artuzo et al., 2017), adicionando o desafio de conciliar a proteção ao meio ambiente e a preservação dos recursos naturais existentes (Anderson et al., 2016).

O Brasil é referência mundial em produtividade e inovação (MAPA, 2020), o Produto Interno Bruto (PIB) do agronegócio foi responsável por 21,4\% do PIB total brasileiro em 2019 (MAPA, 2020), o que demostra a importância do agronegócio na economia do país.

Internamente, as mudanças tecnológicas tem elevado a importância da agricultura, que passa a ser um setor que contribui para o desenvolvimento social, político e econômico de um país (Ayodele et al., 2019), em especial, nos países e regiões com tradição agrária.

A tecnologia aplicada à agricultura, denominada inicialmente como agricultura de precisão, gerou um aumento exponencial na produção agrícola. E o conceito deriva dos primórdios da Revolução Verde, na década de 1960, que introduziu novas técnicas de manejo de solo e a utilização eficiente de fertilizantes (Albergoni e Pelaez, 2007), e evoluiu para o debate da agrobiotecnologia, incorporando ações de proteção do meio ambiente e com a possibilidade de ganhos econômicos para o produtor rural com o incremento da produção agrícola, em especial para suprir as demandas crescentes por alimentos, preservando a segurança alimentar (Artuzo et al., 2017).

A tecnologia como aliada ao agronegócio (Alves et al., 2019) que propicia aumento de produtividade sem a necessidade de ampliação da área cultivável. A administração do agronegócio com a utilização da tecnologia auxilia no controle dos custos de produção, bem como na inserção de novos conhecimentos na área visando aumento de produtividade (Bernardo et al., 2018)

A inovação tecnológica proporcionou vantagem competitiva (Luo et al., 2017), porém existem desafios a serem superados com o aumento no uso da tecnologia pelos produtores rurais e empresas do agronegócio, dentre eles o fosso digital, diferença de acesso aos recursos tecnológicos que pode causar prejuízos (Bowen e Morris, 2019).

A inovação afeta o desempenho do agronegócio, de modo que a tecnologia impacta a produtividade do setor (Ayodele et al., 2019). Mudanças foram iniciadas com o aumento da utilização de novas tecnologias no agronegócio, em especial a partir dos anos de 1970, com o desenvolvimento ferramentas e da adoção de inovações relacionadas a adubos químicos e modificações genéticas em grãos e sementes (Bernardo et al., 2018)

Assim, as novas tecnologias adotadas contribuem para o aumento da produtividade e da eficiência na produção de alimentos. Importante destacar que o investimento em tecnologias e inovação é derivado dos setores econômicas, financeiros, ambientais e sociais de um país (Leite e Batalha, 2016).

Dessa forma, a adoção da agricultura de precisão depende da visualização pelo produtor rural de possibilidade de ganhos econômicos com a adoção de inovações e tecnologias em sua rotina, que proporcionarão aumento da produção e que poderá gerar desenvolvimento local e regional (Artuzo et al., 2017).

A tecnologia auxilia na gestão do negócio com o uso no controle dos custos e no aumento da produção agropecuária (Bernardo et al.,2018). A agricultura de precisão é considerada uma inovação tecnológica que proporciona eficiência no 
processo produtivo e colabora na racionalização dos recursos (Artuzo et al., 2017).

\section{Procedimentos Metodológicos}

A revisão da literatura foi realizada com a utilização da Teoria do Enfoque Metaanalítico Consolidada - TEMAC de Mariano e Rocha (2017) que é fundamentada nas leis da bibliometria, como a Lei de Bradford, Lei de Lokta e Lei do 80/20, possibilitando uma revisão da literatura de forma integradora.

A Lei de Bradford estima o grau de relevância dos periódicos, mostrando quais revistas são mais relevantes e que mais publicaram sobre determinado tema. A Lei de Lokta estima o grau de relevância dos autores, indicando quais autores mais publicaram sobre certo assunto. Além dessas, a Lei 80/20 permite encontrar os $20 \%$ dos documentos que equivalem a 80\% das publicações (Guedes e Borschiver, 2005).

A TEMAC é composta por três fases, a primeira consiste na preparação da pesquisa com a escolha dos descritores que serão aplicados na busca no banco de dados escolhido; a segunda na apresentação e inter-relação dos resultados encontrados; e a última o detalhamento da pesquisa.

A consulta foi realizada no banco de dados da Web of Science, no período de 2016 a 2020, na opção de intervalo personalizado, em todas as línguas e áreas do conhecimento, conjugando simultaneamente os descritores inovação, a tecnologia e o agronegócio. A escolha pelos artigos publicados nos últimos cinco anos em base de periódico reconhecida mundialmente serviu para mostrar o estado da arte.

A preparação iniciou-se com a escolha dos descritores que foram aplicados à pesquisa, que no presente caso foram: agribusiness, innovation e technology. Os resultados obtidos foram apenas 28 artigos de pesquisadores brasileiros, assim, optou-se pela escolha dos descritores $a g i^{*}$, innov* e tech* que proporcionam a ampliação da pesquisa e foram localizados 2.427 artigos, dentre eles, 104 artigos de pesquisadores brasileiros. O período de tempo escolhido foi de 2016 a 2020, na base de dados da Web of Science, abordando todas as áreas do conhecimento e todas as línguas, a fim de mostrar o estado da arte.

A apresentação dos estudos compilados foi realizada com a análise dos artigos mais citados, os periódicos que mais publicam sobre o tema, os países que mais publicaram com a temática, as palavras-chave mais utilizadas, anos de publicação, quantidade de artigos publicados por ano, quantidade de citações anuais e as universidades das quais os pesquisadores fazem parte.

$\mathrm{Na}$ terceira etapa da TEMAC, foram compiladas as principais contribuições dos artigos dos pesquisadores brasileiros mais citados, as contribuições científicas, as palavras-chave mais escolhidas, os periódicos e respectivos fatores de impacto que apresentaram a maior quantidade de publicações e qual a língua escolhida para a escrita científica.

Para a apresentação dos resultados da pesquisa TEMAC, foi utilizado o software VOSviewer, versão 1.6.15, que proporciona a visualização das redes bibliométricas formadas pelas palavras-chave escolhidas pelos autores e pelas instituições que mais publicam na área.

\section{Resultados e Discussão}

Inicialmente foram escolhidos os descritores agribusiness, innovation e technology para a busca no banco de dados da Web of Science. Os resultados da pesquisa mostraram que nos últimos cinco anos foram publicados 1.255 artigos com o tema agronegócio (agribusiness), 64.120 com inovação em geral (innovation) e 295.482 com foco na tecnologia (technology).

Buscando os trabalhos com os descritores agribusiness e innovation simultaneamente, foram encontrados 116 artigos em várias línguas nos últimos cinco anos. No cruzamento dos descritores agribusiness e technology foram localizados 110 artigos. Ao pesquisar os descritores agribusiness, innovation e technology, simultaneamente nos últimos cinco anos, considerando artigos publicados em todas as línguas, resultou em 28 trabalhos científicos, dentre eles, 7 são de autores 
brasileiros.

Em nova pesquisa realizada em janeiro de 2021, objetivando abarcar um maior número de artigos, foram utilizados os descritores agri*, innov* e tech* que proporcionaram ampliar os resultados obtidos na pesquisa para 132.192 com agri*, 162.114 com innov* e 1.300 .947 com tech*. Quando conjugados os descritores agri*com innov*, foram obtidos 4.994 artigos, na junção de agri*com tech*, foram compilados 23.917 e ao utilizar innov*com tech* foram 62.058 trabalhos. A utilização simultânea dos três descritores resultou na localização de 2.427 artigos em todas as línguas e áreas de conhecimento, sendo 104 artigos de pesquisadores brasileiros.

A pesquisa foi centrada na tecnologia e inovação no agronegócio com foco nas contribuições dos 104 artigos científicos dos pesquisadores brasileiros, disponíveis na base de dados da Web of Science nos últimos cinco anos (2016 a 2020), sintetizados na Figura 1.

Figura 1 - Resumo dos resultados da pesquisa na Web of Science.

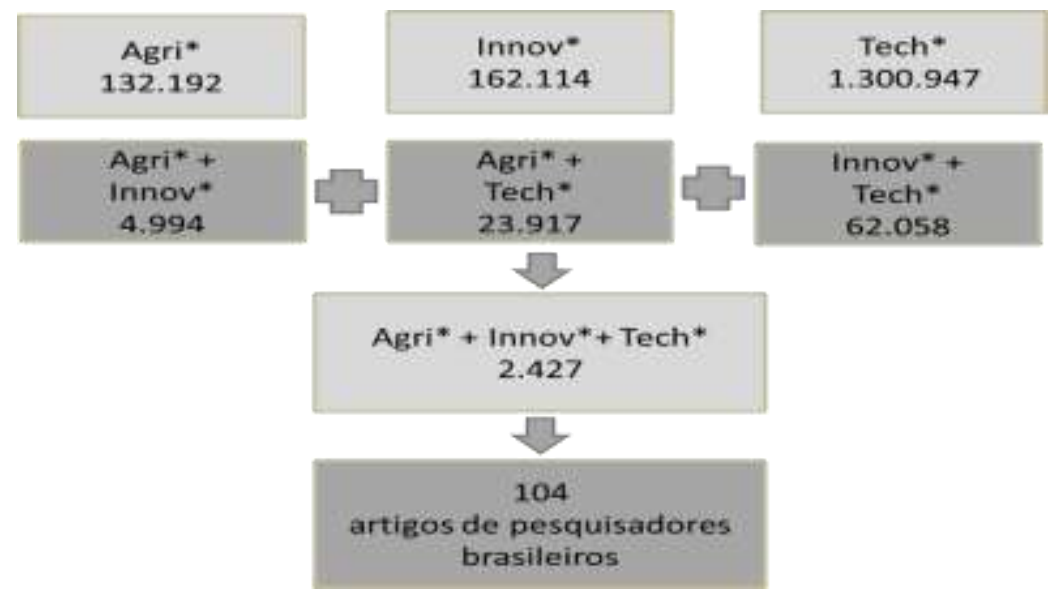

Fonte: Autores (2021).

A quantidade de artigos de pesquisadores brasileiros com a temática foi crescendo ao longo dos anos analisados, iniciando com 15 artigos em 2016 e alcançando 28 no ano de 2020 e a quantidade de citações no mesmo período foi de 5 em 2016 para 262 em 2020, conforme mostra o Quadro 1.

Quadro 1 - Publicações no período de 2016 a 2020.

\begin{tabular}{|c|c|c|}
\hline Ano & $\begin{array}{c}\text { Quantidade de } \\
\text { artigos publicados }\end{array}$ & $\begin{array}{c}\text { Quantidade de } \\
\text { citações }\end{array}$ \\
\hline 2016 & 15 & 5 \\
\hline 2017 & 16 & 38 \\
\hline 2018 & 20 & 117 \\
\hline 2019 & 25 & 193 \\
\hline 2020 & 28 & 262 \\
\hline Total & 104 & 627 \\
\hline
\end{tabular}

Fonte: Dados da Web of Science (2021).

Do total de 104 artigos, apenas 58 apresentaram citações, variando de 171 citações a 1 citação no período de 2016 a 2020, totalizando 627 citações, dentre eles, destacam-se três artigos mais citados no período: Land-use and climate change 
risks in the Amazon and the need of a novel sustainable development paradigm (Nobre et al., 2016) com 171 citações, que representa cerca de 27\% do total; Cooperative membership and dairy performance among smallholders in Ethiopia (Chagwiza et al., 2016) que apresentou 53 citações, cerca de 8\% do total e The role of bioenergy in a climate-changing world (Souza et al., 2017) com 38 citações, totalizando 6\% das citações, em consonância com a lei da bibliometria denominada Lei 80/20, que mostra a importância dos artigos mais citados.

Os cinco periódicos que mais publicaram os artigos, conforme a Lei de Bradford, sobre o tema inovação e tecnologia no agronegócio com a respectiva quantidade de artigos publicados, com o foco e escopo da revista, bem como a classificação Qualis Capes (2013-2016) e o International Standard Serial Number (ISSN) que é o número identificador único para revista e periódicos, relacionados no Quadro 2.

Quadro 2 - Periódicos.

\begin{tabular}{|c|c|c|c|c|c|}
\hline Periódicos & Quantidade & Foco e escopo & $\begin{array}{c}\text { Fator de } \\
\text { impacto (2019) }\end{array}$ & $\begin{array}{c}\text { Qualis Capes } \\
\text { 2013-2016 }\end{array}$ & ISSN \\
\hline $\begin{array}{c}\text { Journal of Cleaner } \\
\text { Production }\end{array}$ & 6 & $\begin{array}{c}\text { Pesquisas e práticas de } \\
\text { produção mais limpa, meio } \\
\text { ambiente e sustentabilidade }\end{array}$ & 7,246 & $\begin{array}{c}\text { A1 } \\
\text { (Administração) }\end{array}$ & 0959-6526 \\
\hline $\begin{array}{l}\text { International Food and } \\
\text { Agribusiness } \\
\text { Management Review }\end{array}$ & 5 & $\begin{array}{c}\text { Prática de gestão na } \\
\text { indústria de alimentos e } \\
\text { agronegócio }\end{array}$ & 1,145 & $\begin{array}{c}\mathrm{A} 2 \\
\text { (Administração) }\end{array}$ & $1559-2448$ \\
\hline $\begin{array}{l}\text { Computers and } \\
\text { Electronics in } \\
\text { Agriculture }\end{array}$ & 3 & $\begin{array}{c}\text { Avanços no } \\
\text { desenvolvimento e aplicação } \\
\text { de hardware de } \\
\text { computador, software, } \\
\text { instrumentação eletrônica e } \\
\text { sistemas de controle para } \\
\text { resolver problemas } \\
\text { na agricultura }\end{array}$ & 3,858 & $\begin{array}{c}\mathrm{A} 2 \\
\text { (Administração) }\end{array}$ & 0168-1699 \\
\hline $\begin{array}{c}\text { Revista Tecnologia e } \\
\text { Sociedade }\end{array}$ & 3 & $\begin{array}{l}\text { Discussão interdisciplinar } \\
\text { das diversas formas de } \\
\text { relação e interação da } \\
\text { tecnologia e sociedade }\end{array}$ & Não disponível & $\begin{array}{c}\text { B3 } \\
\text { (Administração) }\end{array}$ & $1984-3526$ \\
\hline Sustainability & 3 & $\begin{array}{c}\text { Estudos relacionados à } \\
\text { sustentabilidade e ao } \\
\text { desenvolvimento sustentável }\end{array}$ & 2,579 & $\begin{array}{c}\mathrm{B} 1 \\
\text { (interdisciplinar } \\
\text { ) }\end{array}$ & 2071-1050 \\
\hline
\end{tabular}

Fonte: Autores (2021).

O Quadro 2 apresentou os periódicos que publicaram maior quantidade de artigos, já os demais relacionados na base de dados publicaram entre um a dois artigos científicos. Outro ponto importante é relacionar o periódico com o seu fator de impacto que mede o número médio de citações recebidas em um determinado ano por artigos publicados na revista durante os dois anos anteriores.

Os artigos apresentam estudos recentes sobre a tecnologia e inovação no setor do agronegócio em diferentes regiões do mundo e com abordagens específicas, como por exemplo, a análise das mudanças tecnológicas na agricultura, fatores 
determinantes de adoção de tecnologia no meio rural, impacto da agricultura de precisão, os relacionamentos dos produtores com parceiros externos e impactos da acessibilidade ou falta dela no campo.

Dos 2.427 artigos encontrados com a junção simultânea dos três descritores agri*, innov* e tech*, o Brasil apresenta 104 artigos, sendo 81 escritos na língua inglesa, 22 em português e 1 em espanhol, mostrando a tendência da escolha do inglês pela maior possibilidade de visualizações de pesquisadores a nível mundial.

$\mathrm{O}$ artigo com maior quantidade de citações (171 citações), intitulado Land-use and climate change risks in the Amazon and the need of a novel sustainable development paradigm (Nobre et al, 2016), possui como palavras-chave: Amazon tropical forests; Amazon sustainability; Amazon land use; Amazon savannization; climate change impacts. A promoção do desenvolvimento local e regional proposto no trabalho de Nobre et al. (2016) foi objeto de reflexão de Ayodele et al. (2019) que considera a inovação um fator intermediário entre o desempenho da tecnologia e o agronegócio, visando o aumento da produtividade.

Em seguida, o artigo de Chagwiza et al. (2016) denominado Cooperative membership and dairy performance among smallholders in Ethiopia aparece com 53 citações, com destaque para as palavras-chave escolhidas pelos autores: Cooperatives; Dairy producers; Propensity scores matching; Rural poverty; Technological innovation; Ethiopia.

Com o intuito de descrever as principais contribuições dos artigos com maior quantidade de citações, foi elaborado o Quadro 3. Foram selecionados os trabalhos com mais de 20 citações nos últimos cinco anos.

Quadro 3 - Artigos mais citados e suas contribuições científicas.

\begin{tabular}{|c|c|c|c|}
\hline Artigo & Autores & Citações & Contribuições \\
\hline $\begin{array}{l}\text { Land-use and climate change risks in the } \\
\text { Amazon and the need of a novel } \\
\text { sustainable development paradigm }\end{array}$ & $\begin{array}{l}\text { Nobre et al. } \\
\qquad(2016)\end{array}$ & 171 & $\begin{array}{l}\text { Estudo sobre as questões ambientais da Amazônia e de } \\
\text { exploração dos recursos naturais. Os autores sugerem o } \\
\text { uso de inovações, tecnologias digitais e investimento } \\
\text { no capital humano para promover o desenvolvimento } \\
\text { local e regional com vistas à sustentabilidade e } \\
\text { proteção ambiental. }\end{array}$ \\
\hline $\begin{array}{l}\text { Cooperative membership and dairy } \\
\text { performance among smallholders in } \\
\text { Ethiopia }\end{array}$ & $\begin{array}{l}\text { Chagwisa, } \\
\text { Muradian e Ruben } \\
\text { (2016) }\end{array}$ & 53 & $\begin{array}{l}\text { Foi realizada uma análise do impacto de participar ou } \\
\text { não de uma cooperativa de leite na Etiópia. Os } \\
\text { pesquisadores concluíram pela importância da } \\
\text { cooperativa como instituição comercial para promoção } \\
\text { do desenvolvimento rural, transferência de tecnologia e } \\
\text { segurança alimentar. }\end{array}$ \\
\hline $\begin{array}{c}\text { The role of bioenergy in a climate- } \\
\text { changing world }\end{array}$ & $\begin{array}{l}\text { Souza et al. } \\
\text { (2017) }\end{array}$ & 38 & $\begin{array}{l}\text { Os autores abordam as contribuições da produção } \\
\text { sustentável de bioenergia com o uso de inovações e } \\
\text { tecnologias para suprir as necessidades sociais e de } \\
\text { proteção ambiental. }\end{array}$ \\
\hline $\begin{array}{l}\text { Visual soil evaluation: A summary of } \\
\text { some applications and potential } \\
\text { developments for agriculture }\end{array}$ & Ball et al. (2017) & 29 & $\begin{array}{l}\text { A abordagem principal é sobre a utilização de técnica } \\
\text { de avaliação visual do solo para detecção de mudanças } \\
\text { que possam impactar a produção agrícola. A avaliação } \\
\text { é útil para aumentar a conscientização sobre a } \\
\text { qualidade do solo de modo a proporcionar troca de } \\
\text { conhecimentos e ideias para a inovação na agricultura. }\end{array}$ \\
\hline
\end{tabular}




\begin{tabular}{|c|c|c|c|}
\hline $\begin{array}{c}\text { Emerging Agricultural Biotechnologies } \\
\text { for Sustainable Agriculture and Food } \\
\text { Security }\end{array}$ & $\begin{array}{c}\text { Anderson et al. } \\
\text { (2016) }\end{array}$ & 28 & $\begin{array}{c}\text { O artigo apresenta tecnologias emergentes no campo da } \\
\text { biotecnologia agrícola, como feijão e banana } \\
\text { geneticamente modificados, soluções de engenharia } \\
\text { genética, avaliação de ciclo de vida, dentre outras. }\end{array}$ \\
\hline $\begin{array}{c}\text { Assessing the diversity of values and } \\
\text { goals amongst Brazilian commercial- } \\
\text { scale progressive beef farmers using Q- } \\
\text { methodology }\end{array}$ & $\begin{array}{c}\text { Pereira et al. } \\
(2016)\end{array}$ & 23 & $\begin{array}{c}\text { O estudo foi realizado com produtores de carne do } \\
\text { tipos de agricultores, que é relevante, segundo os } \\
\text { pesquisadores, para a adoção da tecnologia no campo. }\end{array}$ \\
\hline $\begin{array}{c}\text { On the need for Innovation in ecological } \\
\text { restoration }\end{array}$ & $\begin{array}{c}\text { Brancalion e } \\
\text { Melis (2017) }\end{array}$ & 20 & $\begin{array}{c}\text { Abordam a importância da inovação ambiental, da } \\
\text { coprodução do conhecimento e dos projetos de } \\
\text { pesquisa nos processos de restauração }\end{array}$ \\
\hline
\end{tabular}

Fonte: Autores (2021).

Os temas abordados nos artigos de pesquisadores brasileiros estão alinhados com a importância da pesquisa no setor do agronegócio para aumento da produtividade e do fomento ao desenvolvimento regional proposto por Moretti (2020).

O uso de palavras-chave nos trabalhos científicos facilita a divulgação científica, permite que os leitores procurem de forma mais simplificada material compatível a partir dos termos de uso em buscas de documentos similares em bancos de dados e na internet, bem como auxiliam o agrupamento dos trabalhos científicos pelos editores e pesquisadores (Harvey, 2012). Dessa forma, a análise das palavras-chave escolhidas pelos autores auxilia outros pesquisadores na busca de material científico e para escolherem as palavras-chave que possibilitem uma maior abrangência do trabalho tanto a nível nacional quanto internacional.

O ponto de partida é a verificação das palavras-chave escolhidas pelos autores nos 104 artigos selecionados que totalizaram 419, mostrando grande diversidade, conforme Figura 2. As palavras-chave mais predominantes foram innovation, agriculture, Brazil, food security, sustentability agriculture e, agribusiness. O agronegócio e a agricultura estão intrinsecamente relacionados à inovação, à tecnologia, sustentabilidade, desenvolvimento sustentável e segurança alimentar, conforme apresenta a Figura 2. 
Research, Society and Development, v. 10, n. 5, e59210515933, 2021

(CC BY 4.0) | ISSN 2525-3409 | DOI: http://dx.doi.org/10.33448/rsd-v10i5.15933

Figura 2 - Palavras-chave escolhidas pelos autores dos 104 artigos selecionados.

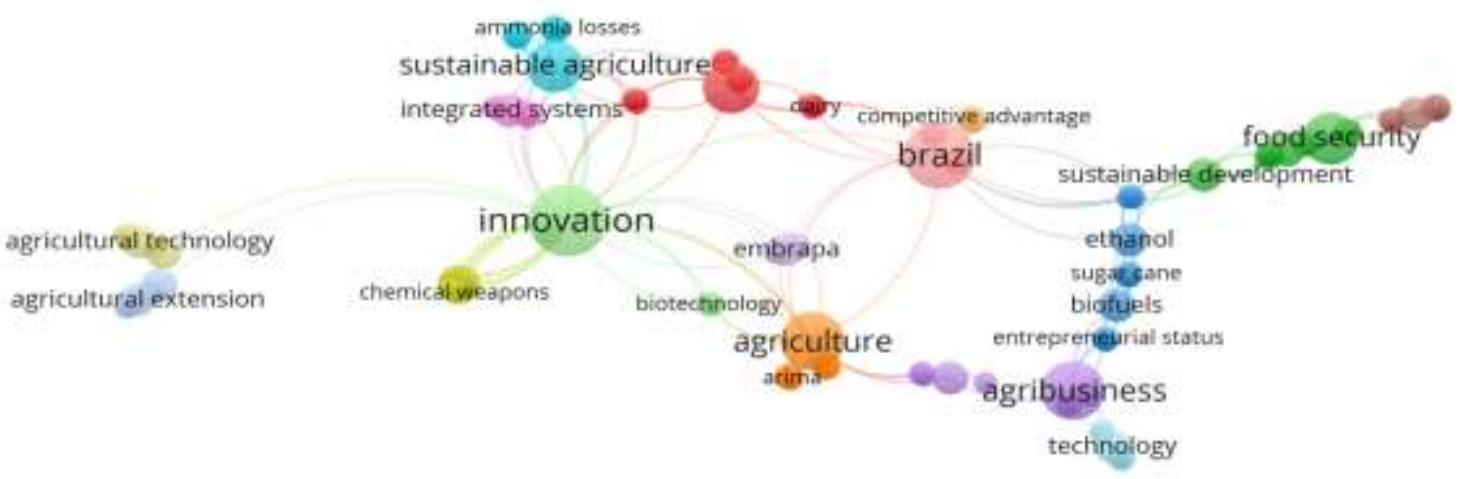

\section{vosviewer}

Fonte: VOSviewer (2021).

As palavras-chave mais utilizadas no período de 2018 a 2020 foram inovação, Brasil, sustentabilidade, agricultura e agronegócio, refletindo a importância da tecnologia no agronegócio em prol do desenvolvimento científico, econômico e social de um país (Ayodele et al.,2019), bem como da proteção ao meio ambiente e da preservação dos recursos naturais existentes (Anderson et al., 2016).

A Figura 3 apresenta as palavras-chave escolhidas pelos autores nos anos de 2018, 2019 e 2020, separadas por cores, variando de azul para 2018 a amarelo para 2020. Em 2020, as palavras-chave mais utilizadas foram etanol, biotecnologia bioeconomia e biogás, laticínio. 
Research, Society and Development, v. 10, n. 5, e59210515933, 2021

(CC BY 4.0) | ISSN 2525-3409 | DOI: http://dx.doi.org/10.33448/rsd-v10i5.15933

Figura 3 - Palavras-chave escolhidas pelos autores dos 104 artigos selecionados.

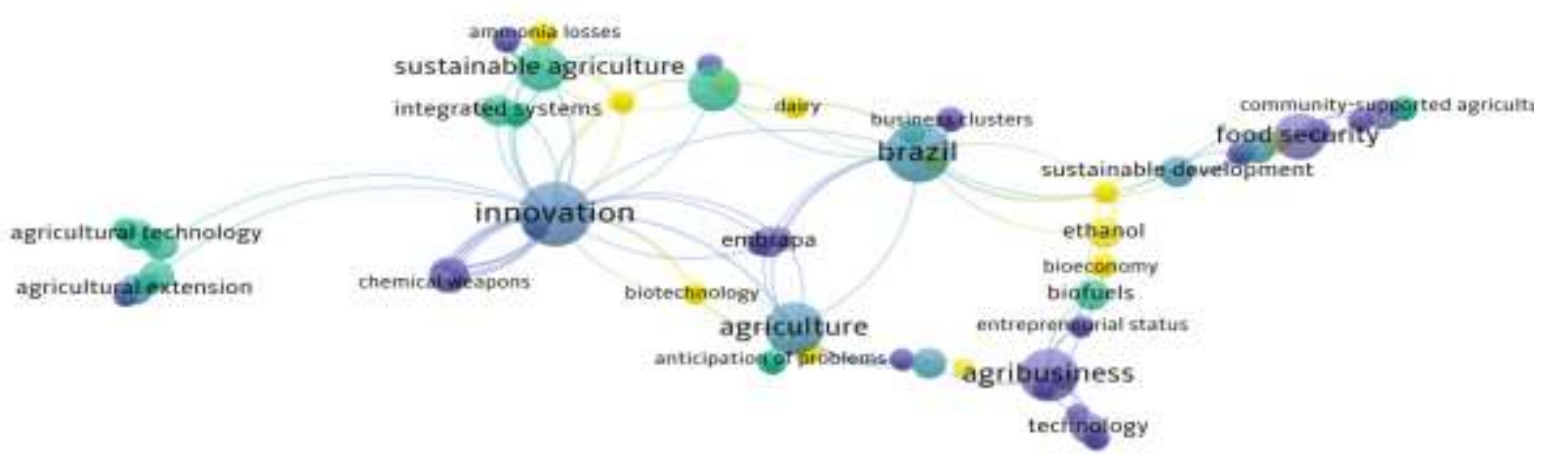

vosviewer

Fonte: VOSviewer (2021).

Os artigos de autores brasileiros que apresentaram mais de 20 citações entre os anos de 2016 a 2020 foram escritos em inglês, cinco deles publicados em periódicos de alto impacto, conforme mostra o Quadro 4.

Quadro 4 - Artigos e palavras-chave.

\begin{tabular}{|c|c|c|c|}
\hline Autores & Palavras-chave escolhidas pelos autores & Periódico & $\begin{array}{l}\text { Fator de impacto } \\
\qquad(2019)\end{array}$ \\
\hline Nobre et al. (2016) & $\begin{array}{l}\text { Amazon tropical forests; Amazon sustainability; Amazon } \\
\text { land use; Amazon savannization; climate change impacts }\end{array}$ & $\begin{array}{l}\text { Proceedings of the } \\
\text { National Academy of } \\
\text { Sciences of the United } \\
\text { States of America }\end{array}$ & 9.412 \\
\hline $\begin{array}{l}\text { Chagwisa, } \\
\text { Muradian e Ruben } \\
\text { (2016) }\end{array}$ & $\begin{array}{c}\text { Propensity scores matching; Rural poverty; Technological } \\
\text { innovation; Ethiopia }\end{array}$ & Food Policy & 4.189 \\
\hline $\begin{array}{l}\text { Souza et al. } \\
\text { (2017) }\end{array}$ & $\begin{array}{c}\text { Bioenergy; Food security; Energy security; Environmental } \\
\text { security; Climate Change; Sustainable } \\
\text { development; Landscapes }\end{array}$ & $\begin{array}{c}\text { Environmental } \\
\text { Development }\end{array}$ & 2,4 \\
\hline Ball et al. (2017) & Soil management; Compaction; VESS & Soil \& Tillage Research & 4.601 \\
\hline $\begin{array}{l}\text { Anderson et al. } \\
\qquad(2016)\end{array}$ & $\begin{array}{l}\text { Agricultural biotechnology, Genetically modified crops, } \\
\text { Sustainability, Food security, Semiochemicals }\end{array}$ & $\begin{array}{c}\text { Journal of Agricultural } \\
\text { and Food Chemistry }\end{array}$ & 4.192 \\
\hline $\begin{array}{l}\text { Pereira et al. } \\
\qquad(2016)\end{array}$ & $\begin{array}{c}\text { Farmer objectives; Farmer typology; Farming } \\
\text { orientations; Technology adoption }\end{array}$ & Agricultural System & 4.212 \\
\hline $\begin{array}{l}\text { Brancalion e Melis } \\
\qquad(2017)\end{array}$ & $\begin{array}{c}\text { Capacity building; cost-effective restoration; restoration } \\
\text { ecology; restoration governance; restoration policy }\end{array}$ & $\begin{array}{l}\text { Annals of the Missouri } \\
\text { Botanical Garden }\end{array}$ & 1.942 \\
\hline
\end{tabular}


Fonte: Autores (2021).

Ao analisar as 269 instituições às quais estão vinculados os autores que publicaram os 104 artigos pesquisados, verifica-se que as três instituições com maior quantidade de publicações foram a Empresa Brasileira de Pesquisa Agropecuária (EMBRAPA) com 19 trabalhos, 13 da Universidade de São Paulo (USP) e 10 da Universidade Estadual Paulista (UNESP). Importante ressaltar que outras intuições públicas e privadas apresentaram de 1 a 3 publicações, porém optou-se por relacionar no Quadro 5 apenas aquelas com 4 ou mais trabalhos científicos com a temática estudada.

Quadro 5 - Instituições, país de origem e quantidade de publicações.

\begin{tabular}{|c|c|c|}
\hline INSTITUIÇÃo & PAÍS & Quant. Publicações \\
\hline Empresa Brasileira de Pesquisa Agropecuária (EMBRAPA) & Brasil & 19 \\
\hline Universidade de São Paulo (USP) & Brasil & 13 \\
\hline Universidade Estadual Paulista (UNESP) & Brasil & 6 \\
\hline Universidade Estadual de Campinas (UNICAMP) & Brasil & 6 \\
\hline Universidade Federal de Santa Catarina (UFSC) & Brasil & 6 \\
\hline Universidade Federal do Rio Grande do Sul (UFRGS) & Brasil & 5 \\
\hline National Research Institute for Agriculture and Food (INRAE) & França & 5 \\
\hline Universidade Federal de Lavras (UFLA) & Brasil & 4 \\
\hline Agricultural Research for Development (CIRAD) & Brança & 4 \\
\hline Universidade de Brasília (UnB) & Brasil & 4 \\
\hline Universidade Federal de São Carlos (UFSCAR) & Holanda & 4 \\
\hline
\end{tabular}

Fonte: Elaborado pelos autores a partir de dados da Web of Science (2021).

Os autores dos artigos formaram parcerias com outros pesquisadores de instituições localizadas em outros países, como França e Holanda. As coautorias formadas pelos pesquisadores das instituições que publicaram os 104 artigos foram ilustradas por meio do software VOSviewer conforme apresenta a Figura 4, que apresentou a formação de quatro clusters. Quanto maior o tamanho do nó na rede, maior a quantidade de artigos com pesquisadores daquele país.

O Brasil ocupa posição de destaque central e possui ligações com os demais países que aparecem na Figura 4, pois a análise foi centrada em artigos que possuam pelo menos um pesquisador brasileiro como autor. 
Figura 4 - Instituições nos 104 artigos.

portugal

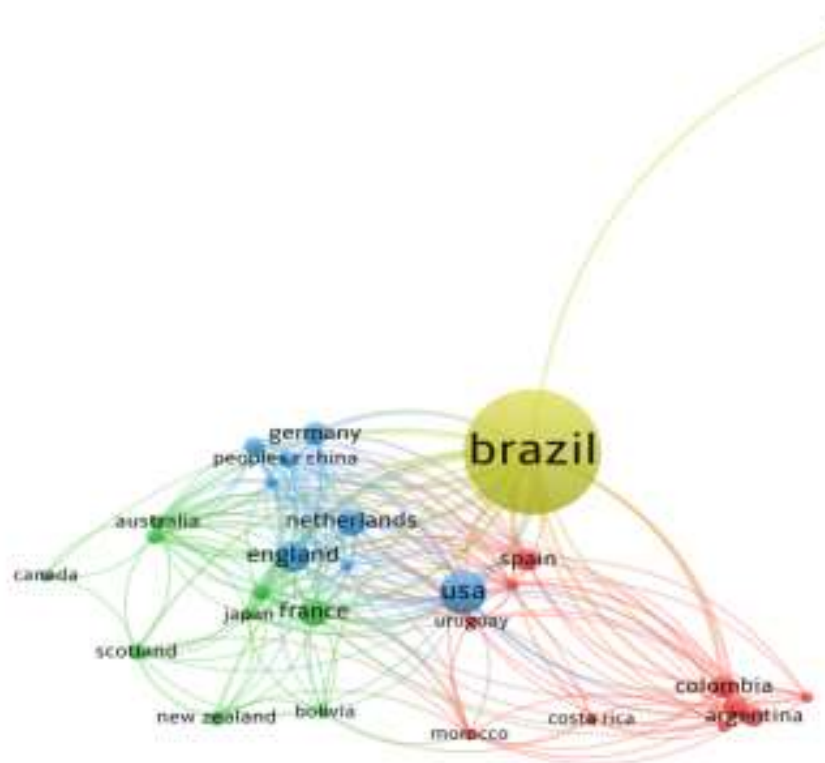

Fonte: VOSviewer (2021).

O banco de dados da Web of Science divide os trabalhos científicos em categorias de assuntos para facilitar a busca pelos pesquisadores. Ao analisar a disponibilização dos 104 artigos nas categorias, considerando a quantidade de artigos publicados igual ou superior a 5, percebe-se que as ciências ambientais possuem a maior quantidade de trabalhos, seguida da agricultura multidisciplinar com 14, Gestão com 11, Estudos Ambientais e Tecnologia Verde Sustentável com 10 cada um e assim por diante, conforme Quadro 6.

Quadro 6 - Quantidade de artigos por categorias da Web Of Science.

\begin{tabular}{|c|c|}
\hline Categorias & Quantidade de artigos \\
\hline Ciências ambientais & 17 \\
\hline Agricultura multidisciplinar & 14 \\
\hline Gestão & 11 \\
\hline Estudos ambientais & 10 \\
\hline Tecnologia sustentável & 9 \\
\hline Política de economia agrícola & 9 \\
\hline Agronomia & 7 \\
\hline Engenharia ambiental & 6 \\
\hline Tecnologia da ciência alimentar & 5 \\
\hline Negócio & 5 \\
\hline Ciências multidisciplinares & 9 \\
\hline
\end{tabular}

Fonte: Web of Science (2021). 
A partir do estado da arte, procurou-se realizar análise do artigo que foi mais citado por ano com a finalidade de apurar qual o assunto e área de pesquisa que mais despertou interesse nos pesquisadores, conforme relacionado no Quadro 7.

Quadro 7 - Artigos mais citados por ano.

\begin{tabular}{|c|c|c|c|c|c|}
\hline Ano & Artigo & Autores & Área de Pesquisa & Citações & Contribuições \\
\hline 2016 & $\begin{array}{l}\text { Land-use and climate } \\
\text { change risks in the Amazon } \\
\text { and the need of a novel } \\
\text { sustainable development } \\
\text { paradigm }\end{array}$ & $\begin{array}{l}\text { Nobre et al. } \\
\text { (2016) }\end{array}$ & Ciência e Tecnologia & 171 & $\begin{array}{c}\text { Defende um novo paradigma de } \\
\text { desenvolvimento sustentável com } \\
\text { investimento em capital humano, a partir } \\
\text { de uma abordagem de inovação de alta } \\
\text { tecnologia. }\end{array}$ \\
\hline 2017 & $\begin{array}{l}\text { The role of bioenergy in a } \\
\text { climate-changing world }\end{array}$ & $\begin{array}{l}\text { Souza et al. } \\
\text { (2017) }\end{array}$ & $\begin{array}{c}\text { Bioenergia; } \\
\text { Segurança Alimentar; } \\
\text { Seguranca energetica; } \\
\text { Segurança Ambiental; } \\
\text { Alterações } \\
\text { Climáticas; } \\
\text { Desenvolvimento } \\
\text { sustentável; Paisagens }\end{array}$ & 38 & $\begin{array}{l}\text { Discute a importância da bioenergia, } \\
\text { descreve os desafios e questões políticas } \\
\text { que envolvem o processo de produção, } \\
\text { realiza recomendações de políticas } \\
\text { públicas para a produção sustentável de } \\
\text { biomassa. }\end{array}$ \\
\hline 2018 & $\begin{array}{l}\text { The paradox of sustainable } \\
\text { innovation: The 'Eroom' } \\
\text { effect (Moore's law } \\
\text { backwards) }\end{array}$ & $\begin{array}{l}\text { Hall el al. } \\
\text { (2018) }\end{array}$ & $\begin{array}{c}\text { Ciência e Tecnologia, } \\
\text { Engenharia e Ciências } \\
\text { Ambientais }\end{array}$ & 13 & $\begin{array}{l}\text { Conclui que existem desafios a serem } \\
\text { enfrentados na inovação tecnológica, tais } \\
\text { como processos de regulação, políticas de } \\
\text { restrição comercial, dentre outros. }\end{array}$ \\
\hline 2019 & $\begin{array}{l}\text { Anaerobic co-digestion of } \\
\text { sweet potato and dairy } \\
\text { cattle manure: A technical } \\
\text { and economic evaluation } \\
\text { for energy and biofertilizer } \\
\text { production }\end{array}$ & $\begin{array}{l}\text { Montoro et } \\
\text { al. (2019) }\end{array}$ & $\begin{array}{c}\text { Ciência e Tecnologia, } \\
\text { Engenharia e Ciências } \\
\text { Ambientais }\end{array}$ & 13 & $\begin{array}{l}\text { Evidencia a importância da inovação no } \\
\text { uso de batata doce conjugada com esterco } \\
\text { para geração de biogás e contribui para o } \\
\text { aumento da sustentabilidade nas } \\
\text { propriedades leiteiras. }\end{array}$ \\
\hline 2020 & $\begin{array}{l}\text { The } 4 \text { p1000 initiative: } \\
\text { Opportunities, limitations } \\
\text { and challenges for } \\
\text { implementing soil organic } \\
\text { carbon sequestration as a } \\
\text { sustainable development } \\
\text { strategy. }\end{array}$ & $\begin{array}{l}\text { Rumpel et } \\
\text { al. (2020) }\end{array}$ & $\begin{array}{c}\text { Engenharia, Ciências } \\
\text { Ambientais e } \\
\text { Ecologia. }\end{array}$ & 20 & $\begin{array}{l}\text { Aborda a mudança climática, segurança } \\
\text { alimentar, ciclo do carbono e práticas } \\
\text { ambientalmente saudáveis. } \\
\text { Os autores descrevem a iniciativa francesa } \\
\text { (4p1000), cujo objetivo é demonstrar que a } \\
\text { agricultura exerce papel importante na } \\
\text { preservação ambiental. }\end{array}$ \\
\hline
\end{tabular}

Fonte: Web of Science (2021).

As contribuições dos autores nacionais foram compiladas com a finalidade de mostrar as áreas de pesquisa, as citações e as principais contribuições de cada artigo científico mais citado por ano, considerando o período de 2016 a 2020. Os resultados mostram que os pesquisadores brasileiros abordam assuntos diferentes, porém com foco na sustentabilidade, usos racionais de recursos naturais, produção de energia limpa, aumento na produtividade no campo, promoção do desenvolvimento sustentável, preservação ambiental e segurança alimentar. 
A partir dos resultados da pesquisa, depreende-se a tendência crescente das publicações em língua inglesa e em parceria com pesquisadores de outros países como estratégia para a divulgação internacional, amplitude de alcance do trabalho científico à sociedade e aumento das chances de citações.

A variedade de assuntos abordados sinaliza um amplo campo de estudos sobre tecnologia e inovação no agronegócio que pode ser explorado. Outro fator relevante é o aumento crescente das citações dos artigos dos pesquisadores brasileiros ao longo do período de 2016 a 2020, reforçando aparentemente o interesse crescente pela temática.

\section{Considerações Finais}

A principal contribuição do artigo foi apresentar os trabalhos científicos de pesquisadores brasileiros que abordam simultaneamente a tecnologia, a inovação e o agronegócio, a partir do banco de dados da Web of Science.

A quantidade de publicações no período de 2016 a 2020, em todas as línguas e áreas do conhecimento está aumentando gradativamente, com destaque para o aumento crescente na quantidade de citações ao longo dos anos, atingindo o ápice em 2020, o que sinaliza um crescente interesse dos pesquisadores na temática.

Os artigos provenientes de pesquisadores brasileiros foram 104 do total de 2.427 trabalhos científicos, sendo que 81 foram escritos em língua inglesa, 22 em língua portuguesa e 1 em espanhol. Os sete artigos mais citados no período estudado, com vinte citações ou mais, foram escritos em inglês, de forma que coaduna com a ideia de gerar maior alcance da pesquisa a nível internacional e aumentar as chances de citação.

A destacar-se a participação de pesquisadores brasileiros que publicam conjuntamente com outros estudiosos de outros países com destaque para os pesquisadores americanos, ingleses, franceses e holandeses ou de instituições diferentes provenientes do Brasil. Segundo os resultados compilados, a EMBRAPA foi a líder de produções científicas na temática, seguida da USP e UNESP.

Os temas abordados nos artigos nacionais apresentaram grande diversidade, com abordagem nas inovações tecnológicas no campo, de pesquisas na área da inovação e tecnologia no agronegócio, porém com foco no aumento da produtividade, tecnologias emergentes, sustentabilidade, produção de energia limpa, segurança alimentar e desenvolvimento sustentável. As categorias com maior destaque para publicação são ciências ambientais, agricultura multidisciplinar, gestão, estudos ambientais e tecnologia sustentável.

Dentre os periódicos que mais publicaram os artigos de pesquisadores brasileiros, destaca-se a revista nacional Tecnologia e Sociedade. As revistas com maior quantidade de publicações na temática no período estudado foram relacionadas neste trabalho para facilitar o acesso àqueles que desejam publicar na área de inovação e tecnologia no agronegócio.

O mapeamento das publicações de pesquisadores brasileiros na base de dados Web of Science, nos últimos cinco anos, apontou o interesse crescente nas questões relacionadas à agricultura sustentável, à tecnologia no campo, à produção de energia limpa, à segurança alimentar e à inovação no agronegócio.

Conforme foi possível verificar, o artigo com maior quantidade de citações (171 no período) que foi elaborado por Nobre et al. (2016) tem como assunto principal a região Amazônica, o que pressupõe o grande interesse internacional pela região. Assim, como contribuição científica, sugere-se a realização de novos estudos na região Norte do Brasil que abordem o agronegócio, a segurança alimentar, geração de energia limpa, inovação no campo e sustentabilidade.

Para estudos futuros, recomenda-se a aplicação da pesquisa em outras bases de dados ou a utilização de outros descritores com a finalidade de ampliação dos temas que abordam as questões relacionadas ao agronegócio, setor que participa com peso do Produto Interno Bruto brasileiro e que é responsável pela produção de alimentos para alimentar a demanda crescente por alimentação. 
Research, Society and Development, v. 10, n. 5, e59210515933, 2021

(CC BY 4.0) | ISSN 2525-3409 | DOI: http://dx.doi.org/10.33448/rsd-v10i5.15933

\section{Referências}

Albergoni, L \& Pelaez, V. (2007). Da Revolução Verde à agrobiotecnologia: ruptura ou continuidade de paradigmas? Revista de Economia, 33 (1), 31-53.

Alves, et al. (2019). Challenges and Innovations in Agribusiness Technology Based Incubators: A case study of Ineagro. Research, Society and Development, $8(5)$, e1985935.

Anderson et al. (2016). Emerging agricultural biotechnologies for sustainable agriculture and food security. Journal of agricultural and food chemistry, 64(2), 383-393.

Ayodele et al. (2019). Innovation as a mediating of relationship between internal and external environment in agribusiness performance. Маркетинг $i$ менеджмент інновацій, 1(1), 196-207.

Artuzo et al. (2017). Agricultura de precisão: inovação para a produção mundial de alimentos e otimização de insumos agrícolas. Revista Tecnologia $e$ Sociedade, 13(29), 146-161.

Ball et al. (2017). Visual soil evaluation: a summary of some applications and potential developments for agriculture. Soil and Tillage Research, 173(1), 114124.

Bernardo et al. (2018). A Produção do Conhecimento no Setor dos Agronegócios. HOLOS, 6(1), 16-33.

Bowen, R \& Morris, W. (2019). The digital divide: Implications for agribusiness and entrepreneurship Lessons from Wales. Journal of Rural Studies, 72(1), 75-84.

Brancalion, P \& Melis, J. (2017). On the Need for Innovation in Ecological Restoration. Annals of the Missouri Botanical Garden, $102(2), 227-236$.

Carayannis et al. (2018). Agri-science to agri-business: the technology transfer dimension. The Journal of Technology Transfer, 43(4), 837-843.

Chagwiza et al. (2016). Cooperative membership and dairy performance among smallholders in Ethiopia. Food Policy, 59(1), 165-173.

Gonçalves et al. (2018). Reflexões atualizadas sobre o contexto do agronegócio brasileiro. Revista Agroalimentaria, 24(46), 89-101.

Guedes, V \& Borschiver, S. (2005). Bibliometria: uma ferramenta estatística para a gestão da informação e do conhecimento, em sistemas de informação, de comunicação e de avaliação científica e tecnológica. Encontro Nacional de Ciência da Informação, 6(1), 18.

Haberli et al. (2017). Understanding the determinants of adoption of enterprise resource planning (ERP) technology within the agri-food context: the case of the Midwest of Brazil. International Food and Agribusiness Management Review, 20(1030), 729-746.

Hall et al. (2018). The paradox of sustainable innovation: the 'Eroom'effect (Moore’s law backwards). Journal of Cleaner Production, $172(1), 3487-3497$.

Harvey, D. (2012). O espaço como palavra-chave. GEOgraphia, 14(28), 8-39.

Leite, A \& Batalha, M. (2016). Agricultura sustentável e cooperativismo: que possíveis links?. Interciência, 1(1), 660-667.

Luo et al. (2017). Technological innovation in agricultural co-operatives in China: Implications for agro-food innovation policies. Food Policy, 73(1), 19-33.

Mariano, A \& Rocha, M. (2017). Revisão da literatura: apresentação de uma abordagem integradora. (1 ed.). AEDEM International Conference.

Ministério da agricultura, pecuária e abastecimento, M.A.P.A. (2020). Ministério da agricultura, pecuária e abastecimento. https://www.gov.br/agricultura/ptbr/assuntos/politica-agricola/todas-publicacoes-de-politica-agricola/agropecuaria-brasileira-em-numeros/abn-06-2020.pdf/view

Montoro et al. (2019). Anaerobic co-digestion of sweet potato and dairy cattle manure: a technical and economic evaluation for energy and biofertilizer production. Journal of Cleaner Production, 226(1), 1082-1091.

Moretti, C. (2020). Investir em pesquisa agropecuária traz retorno para a sociedade brasileira. Política Agrícola, 1(1), 10-20.

Nobre et al. (2016). Land-use and climate change risks in the Amazon and the need of a novel sustainable development paradigm. Proceedings of the National Academy of Sciences, 113(1), 10759-10768.

Pereira et al. (2016). Assessing the diversity of values and goals amongst Brazilian commercial-scale progressive beef farmers using Qmethodology. Agricultural Systems, 144(1), 1-8.

Rumpel et al. (2020). The 4p1000 initiative: Opportunities, limitations and challenges for implementing soil organic carbon sequestration as a sustainable development strategy. Ambio, 49(1), 350-360.

Santos et al. (2020). O Ecossistema Brasileiro de Pesquisa Agrícola: uma trajetória evolucionária em CT\&I. Revista Brasileira de Gestão e Desenvolvimento Regional, 16(2), 79-88.

Souza et al. (2017). The role of bioenergy in a climate-changing world. Environmental development, 23(1), 57-64. 